\title{
THE GELATIN PARTICLE INDIRECT AGGLUTINATION TEST, A MEANS OF SIMPLE AND SENSITIVE SERODIAGNOSIS OF CHAGAS’ DISEASE
}

\author{
Takao Yamashita ${ }^{1}$, Hisami Watanabe ${ }^{2}$, Marisel Maldonado ${ }^{3}$, \\ Maria Angelica Leguizamon ${ }^{3}$, TADAShi WATANABE ${ }^{1}$, \\ Susumu Saito ${ }^{1}$, Takeshi Shozawa ${ }^{4}$, Yoshiya Sato ${ }^{5}$ \\ AND FuJIRO SENDO ${ }^{1}$ \\ Received November 1 1993/Accepted December 101993
}

\begin{abstract}
An indirect agglutination test using a Trypanosoma cruzi antigen-coated gelatin particles was employed to diagnose trypanosomiasis in Paraguay. Results with this test were quite comparable to those obtained with enzyme-linked immunosorbent assay (ELISA). Furthermore, nonspecific reaction to the gelatin particles alone was not found in either acute or chronic infection. This method is more convenient than the ELISA, since the antigen-conjugated particles is stable for at least 1 year at $4^{\circ} \mathrm{C}$ and since the test itself is short and simple to perform and does not require specialized equipment.
\end{abstract}

\section{INTRODUCTION}

Chagas' disease is a chronic parasitosis caused by T. cruzi. It is a serious health problem in Latin America where estimated 16-18 million people are infected and 100 million are at a risk of infection (WHO report, 1991). Acute Chagas' disease is generally characterized by fever, chagoma, blood parasitemia, lymphadenopathy and a low level of antibodies in serum. A few patients die during the acute phase. Some resolve to the chronic phase in which fatal cardiomyopathy, megacolon, megaesophagus and neuropathies may occur despite the relative absence of routinely demonstrable parasites in their blood or tissues (Garry and David, 1992), although high levels of antibodies have been detected (Krettli, and Brener, 1976).

T. cruzi infection is usually diagnosed by serological methods, except in the acute stage, where parasite isolation is feasible. The polymerase chain reaction (PCR) has been applied to the diagnosis of Chagas' disease (Russomando et al., 1992) as have serological tests such as complement fixation (File and
Kent, 1960), immunofluorescence (Camargo, 1966), hemagglutination (Camargo, 1973) and ELISA (File and Kent, 1960). ELISA and PCR have come into more common use because of their greater sensitivity. However, for ELISA and PCR, specialized materials and reagents such as enzyme-coupled antibodies, micro -ELISA reader, DNA synthesizer, analyzer and primer are required. Recentry, however, an indirect agglutination method using a gelatin particles was used in a screening survey of strongyloidiasis by Sato et al., (1990). This test is sensitive, technically simply and can be performed rapidly without specialized equipment or facilities. This makes it useful for screening and serological diagnosis in endemic areas, especially those in which the electric power supply is unstable. In light of this, we attempted to evaluate whether this method could be used instead of ELISA for the serodiagnosis of Chagas' disease in endemic areas.

\section{MATERIALS AND METHODS}

1. Department of Immunology and Parasitology, Yamagata University School of Medicine, Yamagata 990-23, Japan.

2. Department of Medical Zoology, Niigata University School of Medicine, Niigata 951, Japan.

3. Instituto de Investigaciones en Ciencias de la Salud, Universidad Nacional de Asuncion, Paraguay.

4. Japan Medical Cooperation project on Chagas' disease supported by Japan International Cooperation Agency (JICA).

5. Department of Parasitology, and Research Center of Comprehensive Medicine, School of Medicine, University of the Ryukyus, Okinawa 903-01, Japan.

Correspondence address and requires should be addressed to Takao Yamashita. Department of Immunology and Parasitology,

Yamagata University School of Medicine, Yamagata 990-23, Japan. 
Serum samples were obtained from 40 Paraguayan subjects. Informed consent was obtained from all patients before their involvement in this study. Of these 40 subjects, 10 had $T$. cruzi in their blood, 10 exhibited cardiomyopathy or megacolon but no parasites were detected in their blood, 10 showed only positive antibody response against $T$. cruzi antigen by ELISA and 10 were healthy subjects as controls.

\section{T. cruzi antigen}

T. cruzi epimastigotes of RF isolate which was isolated in Paraguay from case of chagas' disease (Mimori et al., 1992) were used to prepare the soluble antigen. Epimastigotes were washed 3 times by centrifugation in phosphate buffered saline (PBS) and then resuspended in PBS containing 1\% TritonX-100 (Wako Chemical Co., Osaka, Japan), 1mM phenylmethylsulfonyl fluoride (Sigma Chemical Co., St Louis, MO) and $10 \%$ glycerol (Wako). The suspension was sonicated 5 times for $30 \mathrm{sec}$ periods, followed by centrifugation at $10,000 \times \mathrm{g}$ for $1 \mathrm{~h}$. The supernatant was used as antigen.

\section{ELISA}

This assay was performed with an ELISA kit as previously reported (Maldonado et al., 1990). Antibody titers were determined as the highest dilution of the test serum which gave an optical density $\geqq 0.8$. Sera showing titers of over 1:20 were estimated to be antibody positive against $T$. cruzi antigen.

\section{Gelatin-particles agglutination test (GPAT)}

The GPAT was performed as previously described (Sato and Ryumon, 1990). Briefly, T. cruzi antigen (200 $\mu \mathrm{g} / \mathrm{ml}$ ) was conjugated to artificial gelatin particles (Fujirebio, Inc, Tokyo, Japan) treated with $10^{-5} \mathrm{M}$ tannic acid. After conjugation of antigen, the gelatin particles were washed four times with $0.6 \%$ inactivated normal rabbit serum (NRS), then lyophilized and kept at $4^{\circ} \mathrm{C}$ until use. For estimation of agglutination titer, the lyophilized antigen-coated particles were resuspended to make a final 1\% suspension in $0.6 \%$ NRS. One drop $(25 \mu \mathrm{l})$ of the antigen-coated particles suspension was mixed in the U-bottomed wells with an equal volume of test serum in serial 2-fold dilutions. After settling at room temprature for $3 \mathrm{~h}$, agglutination patterns in the plates were read according to the results in the previous study (Sato and Ryumon, 1990). The antibody titer was determined as the highest serum dilution giving a positive agglutination pattern. Sera showing agglutination titers of over $1: 16$ were esti- mated to be antibody positive against $T$. cruzi antigen.

\section{RESULTS}

The results of GPAT were compared with those of ELISA using sera of patients who have $T$. cruzi in their blood. As shown in Table 1, all patients were antibody positive by GPAT assessment and showed titers ranging from 1:16 to 1:1024. On the other hand, two acute cases with Romana's sign were shown to be antibody negative by ELISA assessment with a cutoff titer of 1:10.

GPAT and ELISA titers were then examined for 10 patients who were parasite negative in their blood but were found having cardiomyopathy or megacolon based on physical examination. The results, shown in Table 2, demonstrated that all patients were antibody-positive in both GPAT and ELISA. Antibody titers using GPAT were generally higher than those with ELISA.

Following the acute phase, most patients remain serologically positive, but asymptomatic, with the absence of demonstrative parasites in either their blood or tissues (Andrade and Andrade, 1979). Sera from persons having neither T. cruzi in their blood nor any symptoms, but which were antibody positive by ELISA, were examined to further estimate the antibody titer using GPAT. As shown in Table 3, all sera were antibody positive with GPAT and titers were higher than those determined by ELISA in 8 out of 10 samples.

Finally, sera samples from healthy subjects that were antibody negative to $T$. cruzi by ELISA were assessed by GPAT (Table 4). Ten sera used in this study were also antibody negative to $T$. cruzi antigen by GPAT. Furthermore, the gelatin particles used as a

Table 1 Comparison of antibody titers by GPAT and ELISA of sera of T. cruzi positive patients

\begin{tabular}{rll}
\hline \multirow{2}{*}{ Case No. } & \multicolumn{2}{c}{ Titer } \\
\cline { 2 - 3 } & GPAT & ELISA \\
\hline${ }^{*} 2$ & $1: 128$ & $1: 10$ \\
$* 3$ & $1: 64$ & 1,20 \\
$* 4$ & $1: 32$ & $1: 10$ \\
$* * 5$ & $1: 128$ & $1: 20$ \\
$* * 6$ & $1: 128$ & $1: 80$ \\
$* * * 7$ & $1: 1024$ & $1: 160$ \\
$* * * 8$ & $1: 1024$ & $1: 80$ \\
$* * * 9$ & $1: 16$ & $1: 40$ \\
$* * * 10$ & $1: 32$ & $1: 40$ \\
\hline
\end{tabular}

*Acute infection with Romana's sign

**Chronic infection with cardiomyopathy

${ }^{* * *}$ Chronic infection without lesions 
Table 2 Comparison of antibody titers by GPAT and ELISA of sera of patients with lesions but who are parasite negative

\begin{tabular}{cll}
\hline \multirow{2}{*}{ Case No. } & \multicolumn{2}{c}{ Titer } \\
\cline { 2 - 3 } & GPAT & ELISA \\
\hline$* 1$ & $1: 512$ & $1: 320$ \\
$* 2$ & $1: 256$ & $1: 80$ \\
$* 3$ & $1: 128$ & $1: 80$ \\
$* 4$ & $1: 512$ & $1: 160$ \\
$* 5$ & $1: 4096$ & $1: 320$ \\
$* 6$ & $1: 256$ & $1: 40$ \\
$* 7$ & $1: 32$ & $1: 320$ \\
$* 8$ & $1: 64$ & $1: 40$ \\
$* * 9$ & $1: 128$ & $1: 640$ \\
$* * 10$ & $1: 1024$ & $1: 160$ \\
\hline
\end{tabular}

${ }^{*}$ Cardiomyopathy, ${ }^{* *}$ Megacolon

Table 3 Comparison of antibody titers by GPAT and ELISA of sera of subjects without lesions or parasites but who showed a positive antibody response to $T$. cruzi antigen by ELISA

\begin{tabular}{cll}
\hline \multirow{2}{*}{ Case No. } & \multicolumn{2}{c}{ Titer } \\
\cline { 2 - 3 } & GPAT & ELISA \\
\hline 1 & $1: 256$ & $1: 160$ \\
2 & $1: 64$ & $1: 160$ \\
3 & $1: 256$ & $1: 40$ \\
4 & $1: 2048$ & $1: 160$ \\
5 & $1: 128$ & $1: 80$ \\
6 & $1: 32$ & $1: 40$ \\
7 & $1: 512$ & $1: 320$ \\
8 & $1: 128$ & $1: 320$ \\
9 & $1: 512$ & $1: 80$ \\
10 & $1: 512$ & $1: 40$ \\
\hline
\end{tabular}

Table 4 Comparioson of antibody titers by GPAT and ELISA of sera from healthy subjects

\begin{tabular}{ccc}
\hline \multirow{2}{*}{ Case No. } & \multicolumn{2}{c}{ Titer } \\
\cline { 2 - 3 } & GPAT & ELISA \\
\hline 1 & $<1: 16$ & $<1: 20$ \\
2 & $<1: 16$ & $<1: 20$ \\
3 & $<1: 16$ & $<1: 20$ \\
4 & $<1: 16$ & $<1: 20$ \\
5 & $<1: 16$ & $<1: 20$ \\
6 & $<1: 16$ & $<1: 20$ \\
7 & $<1: 16$ & $<1: 20$ \\
8 & $<1: 16$ & $<1: 20$ \\
9 & $<1: 16$ & $<1: 20$ \\
10 & $<1: 16$ & $<1: 20$
\end{tabular}

control did not react with any serum sample used in this experiment.

\section{DISCUSSION}

For diagnosis of Chagas' disease, either the T. cruzi parasite or a serological response to $T$. cruzi antigen must be obtained. Direct detection of T. cruzi in blood is possible during the acute phase but is difficult in patients with lower blood levels of the parasite. To overcome this problem, alternative procedures such as xenodiagnosis and hemoculture are used. Xenodiagnosis, however, is expensive, laborious, and limiting with respect to the length time to get results. In addition, the success rate is as low as $40 \%$ (Minter -Goedbloed et al., 1978). In hemoculture, the blood sample is incubated in an apropriate medium for the growth of a potential parasite (Minter-Goedbloed, 1978) but a long cultivation period is needed and contamination can be a problem. A modern method using DNA probes allows for rapid and direct detection of the parasite in the blood (Gonzalez et al., 1988). However, it is difficult to perform without specialized DNA probes and equipment.

In Chagas' disease, IgM antibody is first produced as a primary response and then IgG antibody appears 20 -40 days after the onset of the acute phase of infection (Schmunis et al., 1980). Many methods have been used to detect these antibodies, including complement fixation (Pereira et al., 1980), hemagglutination (Camargo, 1971), indirect immunofluorescence (Cerisola et al., 1970) and ELISA (Maldonado et al., 1990). These techniques have various advantages and disadvantages based on requirements of special equipment, time required for testing and sensitivity.

GPAT was reported to be a simple and sensitive agglutination test for the screening of strongyloidiasis by Sato (1990). In this study, we confirmed the findings that GPAT is easy to perform and has a sensitivity comparable to ELISA and showed that this method could be used for serological diagnose of Chagas' disease. Furthermore, the antibody titer using GPAT was higher than that with ELISA in $76 \%$ of subjects who were shown to be antibody-positive to T. cruzi antigen. GPAT also requires no specialized equipment or facilities and results can be obtained within about $3 \mathrm{~h}$. In addition, the lyophilized antigen-sensitized carrier particles can be stored at $4^{\circ} \mathrm{C}$ for a long period, at least a year or more, without deterioration of the antigen. Moreover, the serum samples can be used without prior absorption by the gelatin particles because these non- 
sensitized particles did not react with the 40 samples used in these experiments. The coloured gelatin particles were also found to be convenient for reading the agglutination pattern. Therefore, GPAT should prove to be useful for serodiagnosis of this disease, both in the laboratories as well as in the field.

In Latin America, distributions of T. cruzi and Leishmania infection often overlap. T. cruzi antigen has been shown to cross react with sera from leishmaniasis patients (Chaffee, 1956; Gam and Neva, 1977; Chiller et al., 1990). In view of this, when parasitological examination is negative and symptoms are not characteristic of recent $T$. cruzi infection but the serological test is positive, there may exist the possibility of leishmaniasis or double infection. We examined ten serum samples from subjects without parasites or lesions. These sera were antibody positive to $T$. cruzi - antigen by GPAT and ELISA. However, it is still difficult to conclude whether these subjects had previous T. cruzi and/or Leishmania-infections. In the future, we will have to provide the specific antigen from $T$. cruzi and develop the specific sero-diagnosis for Chagas' disease.

\section{ACKNOWLEDGMENTS}

We would like to thank Fujirebio Inc., Tokyo, Japan (Mr. S. Hanzawa and Mr. Y. Nagafuchi) for supplying the gelatin paticless. We also thank Drs. M.A. Cabello, A.R. Arias and R.M. Azorero, Instituto de Investigaciones en Ciencias de la Salud, Asuncion, Paraguay, for their help in providing serum samples from patients with Chagas' disease. This study was performed as part of a Paraguay/Japan medical cooperation project on Chagas' disease supported by the Japan International Cooperation Agency (JICA).

\section{REFERENCES}

$1)$ Andrade, Z. and Andrade, S.G. (1979): Patologia. In Trypanosoma cruzi e Doenca de Chagas. Z. Brener and Z. Andrade, eds. Guanabara Koogan, Rio de Janeiro, P. 199

2 ) Camargo, M.E. (1966): Fluorescent antibody test for the serodiagnosis of American trypanosomiasis. Technical modification employing preserved culture forms of Trypanosoma cruzi in a slide test. Rev. Inst. Med. Trop. Sao Paulo, 8, 227-234

3 ) Camargo, M.E., Hoshino, S. and Siqueira, G.R.V. (1973): Hemagglutination with preserved, sensitized cells, a practial test for routine serologic diagnosis of American trypanosomiasis. Rev. Inst. Med. Trop. Sao Paulo, 15, 81 $-85$
4) Chaffee, E.F., Fife, E.H. and Kent, J.F. (1956): Diagnosis of Trypanosoma cruzi infection by complement fixation. Am. J. Trop. Med. Hyg., 5, 763-771

5 ) Chiller, T.M., Samudio, M.A. and Zoulex, G. (1990): IgG antibody reactivity with Trypanosoma cruzi and Leishmania antigens in sera of patients with Chagas' disease and leishmaniasis. Am. J. Trop. Med. Hyg., 43, 650-656

6 ) Fife, E.H. and Kent, J.F. (1960): Protein and carbohydrate complement fixing antigens of Trypanosoma cruzi. Am. J. Trop. Med. Hyg., 9, 512-517

7 ) Gam, A.A. and Neva, F.A. (1977): Comparison of cell culture with epimastigote antigens of Trypanosoma cruzi. Am. J. Trop. Med. Hyg., 26, 47-57

8 ) Takle, G.B. and David, S. (1992): 11 South American trypanosomiasis (Chagas' disease). In: Immunology and molecular biology of parasitic infections, Kenneth S. Warren (editors). Boston: Blackwell Scientific Publications, pp 213-236

$9)$ Gonzalez, A., Prediger, E., Huecas, M. E., Nogueria N. and Lizardi PM. (1984): Minichromozomal repeptitive DNA in Trypanosoma cruzi: its use in a high-sensitivity parasite detection assay. Proc. Natl. Acad. Sci. USA, 81, $3356-3360$

10) Krettli, A.V. and Brener, Z. (1976): Protective effects of specific antibodies in Trypanosoma cruzi infections. J. Immunol. 116, 755-760

11) Maldonado, M., Ichinose, Y., Samudio, M., Arias, A.R., Sakamoto, M., Azorero, R.M. and Kanbara, H. (1990): Application of two typs of Trypanosoma cruzi amastigotes of different virulence to ELISA for Chagas' disease. Jpn. J. Trop. Med. Hyg., 18, 325-332

12) Mimori, T., Maldonado, M., Samudio, M., Arias, A. R., Moreno, R. and Sakamoto, M. (1992): Characterization of Trypanosoma cruzi isolates from Paraguay, using restriction enzyme analysis of kinetoplast DNA. Ann. Trop. Med. Parasit., 86, 231-237.

13) Minter-Goedbloed E. (1978): The primary isolation by haemoculture of Trypanosoma (Schizotrypanum) cruzi from animals and man. Transactions of the Royal Society of Tropical Medicine and Hygiene 72, 22-30

14) Russomando, G., Figueredo, A., Almiron, M., Sakamoto, M. and Morita, K. (1992): Polymerase chain reaction -based detection of Trypanosoma cruzi DNA in serum. J. Clin. Microbiol. 30, 2864-2868

15) Sato, Y. and Ryumon, I. (1990): Gelatin particle indirect agglutination test for serodiagnosis of human strongyloidiasis. Jpn. J. Parasitol., 39, 213-219

16) Schmunis, G.A., Szarfman, A., Coarasa, L., Guilleron, C. and Pelalta, J.M. (1980): Anti-Trypanosoma cruzi agglutinins in acute human Chagas' disease. Am. J. Trop. Med. Hyg., 29, 170-178

17) World Health Organization (1991): Control of Chagas disease. Geneva: WHO Technical Report Series, 811, 1 $-95$ 